\title{
Intake of Koji Amazake Improves Defecation Frequency in Healthy Adults
}

\author{
Atsushi Kurahashi ${ }^{1, *(\mathbb{D}}$, Toshihiko Enomoto ${ }^{1}$, Yoshifumi Oguro ${ }^{1}$, Ayana Kojima-Nakamura ${ }^{1}$, Kazuya Kodaira ${ }^{1}$, \\ Kenichi Watanabe ${ }^{2}$, Nobuhiro Ozaki ${ }^{3}$, Hiroshi Goto ${ }^{4}$ and Masao Hirayama ${ }^{4}$ \\ 1 Hakkaisan Brewery Co., Ltd., 1051 Nagamori, Minamiuonuma City, Niigata 949-7112, Japan; \\ toshihiko.enomoto@hakkaisan.jp (T.E.); y.oguro@hakkaisan.jp (Y.O.); \\ ayana.nakamura@hakkaisna.co.jp (A.K.-N.); k.kodaira@hakkaisan.jp (K.K.) \\ 2 Department of Endocrinology and Metabolism, Niigata University Graduate School of Medical and Dental Sciences, \\ 754 Ichibancho, Asahimachi-dori, Chuo-ku, Niigata City, Niigata 951-8510, Japan; wataken@med.niigata-u.ac.jp \\ 3 Niigata Medical Association of Occupational Health Inc., 1185-3 Kitaba, Nishi-ku, Niigata City, \\ Niigata 950-1187, Japan; tomiyama@niwell.or.jp \\ 4 Niigata Bio-Research Park Inc., 316-2 Higashijima, Akiha-ku, Niigata City, Niigata 956-0841, Japan; \\ goto@nbrp.co.jp (H.G.); hirayama@nbrp.co.jp (M.H.) \\ * Correspondence: a.kurahashi@hakkaisan.jp; Tel.: +81-25-788-0910
}

\section{check for} updates

Citation: Kurahashi, A.; Enomoto, T.; Oguro, Y.; Kojima-Nakamura, A.; Kodaira, K.; Watanabe, K.; Ozaki, N.; Goto, H.; Hirayama, M. Intake of Koji Amazake Improves Defecation Frequency in Healthy Adults. J. Fungi 2021, 7, 782. https://doi.org/ 10.3390/jof7090782

Academic Editors:

Katsuhiko Kitamoto and Yujiro Higuchi

Received: 1 September 2021

Accepted: 18 September 2021

Published: 21 September 2021

Publisher's Note: MDPI stays neutral with regard to jurisdictional claims in published maps and institutional affiliations.

Copyright: (c) 2021 by the authors. Licensee MDPI, Basel, Switzerland. This article is an open access article distributed under the terms and conditions of the Creative Commons Attribution (CC BY) license (https:/ / creativecommons.org/licenses/by/ $4.0 /)$.

\begin{abstract}
Reportedly, the intake of koji amazake, a beverage made from steamed rice fermented by Aspergillus oryzae, improves defecation frequency. However, its functional ingredients and mechanism of action remain unclear. To compare the effects of koji amazake and a placebo beverage on defecation frequency and to identify the functional ingredients and mechanism of action, a randomized, placebocontrolled, double-blind parallel-group comparative trial was performed on two groups. The koji amazake had $302 \pm 15.5 \mathrm{mg} / 118 \mathrm{~g}$ of $A$. oryzae cells, which was not in the placebo. Compared with the placebo group, the koji amazake group showed a significant increase in weekly defecation frequency at 2 weeks (5.09 days vs. 4.14 days), 3 weeks (5.41 days vs. 4.18 days), and 4 weeks (5.09 days vs. 3.95 days), along with an increase in the weekly fecal weight at 4 weeks (724 g vs. $501 \mathrm{~g}$ ). The intake of koji amazake did not induce significant intergroup differences in the fecal SCFA concentration, whereas it significantly decreased the relative abundance of Blautia and significantly increased that of Bacteroides at 3 weeks. Therefore, koji amazake intake improved defecation frequency, and A. oryzae cells played potentially important roles as functional ingredients.
\end{abstract}

Keywords: amazake; Aspergillus oryzae; defecation; intestinal microbiota; koji

\section{Introduction}

Several fermented beverages and food products consumed globally are produced by the action of microorganisms and/or their enzymes. Fermented foods form a significant part of diets worldwide and typically constitute approximately one-third of the global food intake [1]. Fermentation not only improves food preservation, nutritional value, and quality, but also benefits human health by facilitating the growth of probiotic strains in foods. Probiotics are viable microorganisms that beneficially affect their hosts, including humans, by improving their intestinal microbial balance [2]. One of the other expected functions of probiotics is the improvement of defecation frequency. Yogurt, a representative fermented food product, contains lactic acid bacteria and bifidobacteria, which reportedly improve defecation frequency in healthy individuals. The intake of live Lactobacillus casei Shirota and L. brevis KB290 improved the defecation frequency and stool quality by enhancing short-chain fatty acid (SCFA) production by intestinal microflora $[3,4]$. A similar effect was reported for Bifidobacterium longum BB536 [5]. Probiotics for improving human defecation have mostly been limited to lactic acid bacteria and bifidobacteria, although a few other food microorganisms have also been considered. 
Recently, two human studies reported the effect of koji amazake on stool defecation. koji amazake is a non-alcoholic, white colored, Japanese traditional sweet beverage made from steamed rice fermented by Aspergillus oryzae [6]. The raw materials are rice-koji and water. It becomes sweetened when saccharification enzymes such as $\alpha$-amylase and glucoamylase of $A$. oryzae break down rice starch in rice-koji into glucose in a saccharification process at 50-60 ${ }^{\circ} \mathrm{C}$. Therefore, koji amazake includes rice, A. oryzae, and its metabolites. In one study, the defecation frequencies of female college students $(n=12)$ in koji amazake intake and non-intake groups were compared, and a statistically significant difference was found in the intragroup comparison from baseline to 12 days post-intake [7]. The second study showed that in the comparison of defecation frequencies between two groups with the intake of koji amazake and its mixture with Lactobacillus sakei UONUMA, the defecation frequency was significantly higher in the koji amazake group [8]. However, both reports lacked scientific evidence, including information on the functional ingredients and mechanism of action underlying the increase in defecation frequency, which are essential parameters for the Japanese approval system of Foods for Specified Health Use [9]. Probiotics are widely known to improve gastrointestinal health through the following mechanisms: modification of gastrointestinal microbiota, production of probiotic metabolites, including prebiotics, and production of SCFAs [10]. Prebiotics and probiotics are known to increase the defecation frequency [11]. Prebiotics are non-digestible food ingredients, such as non-digestible fiber and oligosaccharides, with beneficial effects, such as selective stimulation of colonic bacterial species [12].

We have previously reported that koji amazake contains over 300 compounds, including the fermentation metabolites of $A$. oryzae [13]. In particular, koji amazake contains various glucooligosaccharides, such as maltose, nigerose, kojibiose, trehalose, sophorose, gentiobiose, maltotriose, isomaltotriose, panose, and raffinose [14]. These glucooligosaccharides contain prebiotic components, such as gentiobiose (Glc( $\beta 1-6)$ Glc) [15], raffinose (Gal $(\alpha 1-$ 6) Glc( $\beta 1-2) \mathrm{Fru})[16]$, isomaltooligosaccharides (IMOs) (including isomaltose (Glc $(\alpha 1-6) \mathrm{Glc})$, isomaltotriose ( $\alpha 1-6) \mathrm{Glc}(\alpha 1-6) \mathrm{Glc})$, and panose $(\mathrm{Glc}(\alpha 1-6) \mathrm{Glc}(\alpha 1-4) \mathrm{Glc}))$, which reportedly modify the intestinal microbial composition and improve gastrointestinal conditions [17,18]. In addition, rice-koji, the raw material for koji amazake, contains monohexosylceramides composed of two sugar moieties: $N-2^{\prime}$-hydroxyoctadecanoyl-1-O- $\beta$-D-glucopyranosyl-9methyl-4,8-sphingadienine and $N$-2' - hydroxyoctadecanoyl-1-O- $\beta$-D-galactopyranosyl-9methyl-4,8-sphingadienine [19-21]. Reportedly, these koji ceramides serve as prebiotics for Blautia coccoides [22]. koji amazake also contains A. oryzae cells. It has been reported that chitin-glucan prepared from the cell wall of Aspergillus niger induces specific changes in intestinal bacteria and further increases the bacterial metabolites in feces, including butyric, iso-valeric, caproic, and vaccenic acids [23]. Therefore, koji amazake fulfills the required conditions for classification as a synbiotic, which contains multiple potential prebiotic candidates for improving defecation frequency. However, only a limited number of studies are available on the functional ingredients and mechanisms of action.

This was a randomized, placebo-controlled, double-blind parallel-group comparative trial, in which rice syrup was used as a placebo. The primary outcome was the defecation frequency per week, and secondary outcomes were changes in fecal characteristics, fecal SCFA content, and fecal microbiota composition. The information obtained was used to determine the active functional ingredients of koji amazake and the mechanism of action.

\section{Materials and Methods}

\subsection{Test Beverages}

Commercial koji amazake (Hakkaisan Brewery Co., Ltd., Minamiuonuma, Japan) and rice syrup (prepared using hydrolyzing enzymes without fermentation with A. oryzae, as previously described [14]) (118 g/bottle) were used as the test beverages. The nutritional content per bottle of koji amazake and placebo are shown in Table 1. The energy, protein, lipid, carbohydrate, available carbohydrate, dietary fiber, ash, and moisture contents were 
measured using the official method adopted by the Japan Food Research Laboratories (Tokyo, Japan), as previously described [24].

Table 1. Nutritional values of test beverages (per $118 \mathrm{~g}$ in a bottle).

\begin{tabular}{|c|c|c|}
\hline Item & koji amazake & Placebo \\
\hline Energy (Kcal) & 127.4 & 128.6 \\
\hline Moisture (g) & 86.4 & 85.6 \\
\hline Protein $(\mathrm{g})$ & 1.4 & 1.5 \\
\hline Fat $(\mathrm{g})$ & 0.4 & 0.2 \\
\hline Total GlcCer (mg) & 1.39 & 0.41 \\
\hline GlcCer from A. oryzae (mg) & 1.16 & ND \\
\hline GlcCer from rice (mg) & 0.23 & 0.41 \\
\hline Ash (g) & 0.1 & 0.1 \\
\hline Carbohydrate $(\mathrm{CHO})(\mathrm{g})$ & 29.7 & 30.6 \\
\hline Dietary fiber $(\mathrm{g})$ & 0.2 & 0.1 \\
\hline Available CHO (g) & 29.5 & 30.5 \\
\hline Digestible CHO (g) & 26.4 & 28.0 \\
\hline Glucose $(\mathrm{g})$ & 26.1 & 27.8 \\
\hline Maltose (g) & 0.17 & 0.20 \\
\hline Trehalose (g) & 0.14 & ND \\
\hline Prebiotics $(\mathrm{g})$ & 3.12 & 2.47 \\
\hline Isomaltose $(\mathrm{g})$ & 1.96 & 1.89 \\
\hline Isomaltotiose (g) & 0.10 & 0.18 \\
\hline Panose (g) & 0.10 & ND \\
\hline Sophorose (g) & 0.66 & 0.32 \\
\hline Nigerose (g) & 0.20 & 0.08 \\
\hline Kojibiose (g) & 0.10 & ND \\
\hline
\end{tabular}

\subsection{Quantification for A. oryzae and Its Metabolites in Koji Amazake}

Contents of oligosaccharides, glycosylceramides (GlcCer), and A. oryzae cells were quantified in order to reveal functional components in koji amazake. The levels of glucose and other saccharides were measured as previously described $(n=3)[13,14]$.

The standard GlcCer from A. oryzae was prepared at a facility at the Foundation for Promotion of Material Science and Technology of Japan (Tokyo, Japan). Ten grams of freeze-dried A. oryzae mycelium was homogenized with $250 \mathrm{~mL}$ of methanol and then with $500 \mathrm{~mL}$ of chloroform. Next, $200 \mathrm{~mL}$ of water was added, and the mixture was filtered for 1 min using a filter paper with a separating funnel and then allowed to stand overnight. The organic solvent phase was dried using an evaporator; $10 \mathrm{~mL}$ of $\mathrm{KOH}-m e t h a n o l ~ s o-$ lution, $0.5 \mathrm{~mL}$ of chloroform/methanol $(2: 1, v / v)$, and $7.5 \mathrm{~mL}$ of water were added; and a saponification was performed for $3 \mathrm{~h}$ at $37^{\circ} \mathrm{C}$. The saponified sample was mixed with $16 \mathrm{~mL}$ of chloroform, $8 \mathrm{~mL}$ of methanol, and $6 \mathrm{~mL}$ of water. The extraction process described above was performed three times. Next, the organic solvent phase was collected and evaporated. The dried sample was dissolved in methanol and was then subjected to silica gel chromatography. The sample was washed using chloroform/methanol (99:5, $v / v$ ). Afterwards, a fraction containing GlcCer was eluted using chloroform/methanol $(90: 10, v / v)$ and evaporated. The lipid fraction containing GlcCer was separated precisely using an HPLC system equipped with a fraction collector to obtain two species of GlcCer, which were used as GlcCer standards for A. oryzae. GlcCer from rice was purchased as a commercial product (Nagara Science, Gifu, Japan). The GlcCer content in koji amazake and placebo was measured using ACQUITY UPLC H-Class with ACQUITY QDa Detector (Waters Corporation, Milford MA, USA). GlcCer was separated using the ACQUITY UPLC BEH C18 column $(2.1 \mathrm{~mm} \times 150 \mathrm{~mm}, 1.7 \mu \mathrm{m})$ (Waters Corporation, Milford MA, USA). Solvent A was $10 \mathrm{mM}$ ammonium formate, and solvent $\mathrm{B}$ was $10 \mathrm{mM}$ ammonium formatemethanol. The duality mobile phase gradient used for analysis was as follows: $0 \mathrm{~min}, 3 \% \mathrm{~A}$, $97 \% \mathrm{~B} ; 15-19 \min 100 \% \mathrm{~B} ; 20-25 \mathrm{~min} 3 \% \mathrm{~A}, 97 \% \mathrm{~B}$. The flow rate was $0.2 \mathrm{~mL} / \mathrm{min}$. The other 
parameters adopted were as follows: column temperature, $40^{\circ} \mathrm{C}$; injection volume $1 \mu \mathrm{L}$. Each GlcCer content of koji amazake $(n=17)$ and placebo $(n=3)$ is shown in Table 1 .

With slight modification from a previous study [25], the amount of $A$. oryzae cells in koji amazake $(n=17)$ and placebo $(n=3)$ was estimated by quantifying $\mathrm{N}$-acetylglucosamine (GlcNAc). Beverage sample ( $5 \mathrm{~g}$ ) was centrifuged and supernatant was discarded. The pellet was washed 3 times with $50 \mathrm{mM}$ phosphate buffer ( $\mathrm{pH}$ 7.0). In order to degrade chitin in the sample, $10 \mathrm{~mL}$ of $50 \mathrm{mM}$ phosphate buffer containing $10 \mathrm{mg}$ Yatalase (Takara Bio Inc, Japan) was added to the pellet, and then incubated for $1 \mathrm{~h}$ at $37^{\circ} \mathrm{C}$. Quantification of GlcNAc was followed by the method proposed by Reissig et al. [26]. The reaction mix containing $500 \mu \mathrm{L}$ of the sample and $100 \mu \mathrm{L}$ of $0.8 \mathrm{M}$ potassium tetraborate (pH 9.0) was boiled for $3 \mathrm{~min}$ at $100{ }^{\circ} \mathrm{C}$ and then quickly cooled. The $p$-dimetylaminobenzaldehyde (DMAB) solution (10 g of DMAB was dissolved $12.5 \mathrm{~mL} 12 \mathrm{~N} \mathrm{HCl}$ and adjusted to $100 \mathrm{~mL}$ by adding acetic acid) was diluted 9-fold with acetic acid. Cooled sample was mixed with $3 \mathrm{~mL}$ of diluted DMAB solution and incubated for $20 \mathrm{~min}$ at $37^{\circ} \mathrm{C}$. After the sample was cooled, absorbance was measured at $585 \mathrm{~nm}$. In this study, $1 \mathrm{mg}$ mycelium was estimated to be $139 \mu \mathrm{g}$ GlcNAc based on a previous report [25].

\subsection{Study Population}

From 60 applicants, 44 healthy adults with $2-5$ days of weekly defecation were recruited at the Niigata Bio-research Center. The adults were screened according to the inclusion and exclusion criteria. Individuals with screening test results and questionnaire results appropriate for this study, as judged by an investigator, were included. The exclusion criteria were as follows: (i) individuals with diabetes and under medical treatment; (ii) individuals with diseases (e.g., liver, kidney, heart, blood diseases, and infections requiring notification); (iii) individuals with a history of gastrectomy, enterectomy, and gastrointestinal diseases, judged to be inappropriate for this study; (iv) pregnancy, women with chances of pregnancy, and lactating women; (v) participants in other clinical trials; and (vi) individuals with concerns judged to be inappropriate for this study by an investigator.

\subsection{Study Design}

The study had a randomized, placebo-controlled, double-blind parallel-group comparative design. It was conducted between October 2019 and May 2020 at the Niigata Bio-research Center (Niigata, Japan). Randomization was performed in a stratified manner based on the weekly defecation day (days/week) at the screening visit. The study period spanned 6 weeks, consisting of a non-intake observation period ( -1 to $0 \mathrm{w}$ ), intake period $(0$ to $3 \mathrm{w}$ ), and follow-up period ( 4 to $5 \mathrm{w}$ ). The test beverage was administered once daily during the intake period. The participants were instructed to avoid the consumption of other amazake drinks and similar beverages and the introduction of changes to their daily activities, including diet and exercise. After the participants provided written informed consent and their screening procedures were completed, they were recruited to the study. The participants were randomly assigned to two groups: koji amazake and placebo.

During the study, all participants were instructed as follows: avoid an irregular lifestyle, including overeating and poor sleep; avoid introducing changes to routine lifestyle, including diet, sleep, and exercise; do not donate blood; do not start intake of new health foods; and contact the study staff if experiencing any signs or symptoms of changes in health conditions. During the test period starting from the day before testing, the participants were prohibited from drinking alcohol, over-exercising, overeating, and sleeping insufficiently on the day before testing. From 20:00 h on the day before testing, the consumption of food and beverages, except water, was prohibited.

\subsection{Physiological and Biochemical Variables of the Participants}

The following physiological and biochemical variables of the participants were measured during the screening visit using standard methods: height; body weight; body mass index; body fat percentage; systolic and diastolic blood pressure; levels of aspartate 
aminotransferase, alanine aminotransferase, $\gamma$-glutamyl transpeptidase, high-density and low-density lipoprotein cholesterol, triglyceride, blood urea nitrogen, creatinine, fasting blood glucose, white blood cells, red blood cells, hematocrit, hemoglobin, and platelets. These measurements were performed at the Clinical Laboratory Division of the Association of Occupational Health, Inc. (Niigata, Japan).

\subsection{Questionnaires on Defecation and Collection of Fecal Samples}

We instructed the participants to fill out questionnaires on their defecation patterns and characteristics and to collect their fecal samples during three different periods. Questions were asked on the defecation frequency (number of days), defecation frequency (number of times), fecal quantities (in terms of the number of Japanese standard chicken eggs, shown schematically), and fecal characteristics (in terms of shape, color, odor, and sensation after defecation). The participants observed their fecal characteristics, recorded the scores each day throughout the 6-week study period, and submitted the weekly records to the study staff. Each fecal sample was collected by the participants in a designated container from the TechnoSuruga Laboratory Co., Ltd. (Shizuoka, Japan) on the last day of the non-intake observation period $(0 \mathrm{w})$, intake period $(3 \mathrm{w})$, and follow-up period $(5 \mathrm{w})$. The samples were delivered to the TechnoSuruga Laboratory under refrigeration within $24 \mathrm{~h}$ of defecation.

\subsection{Defecation Patterns and Fecal Characteristics}

Using the data collected from the questionnaires, the defecation frequency (number of days/week and number of times/week), fecal weight (g/week), and fecal characteristic scores (for shape, color, odor, and sensation after defecation) for each week were calculated during the 6 weeks of the study period. The fecal weight was estimated by counting the number of Japanese standard chicken eggs ( $50 \mathrm{~g}$ ) approximately representative of the fecal volume, as reported by Ogata et al. [27], and a fecal specific gravity of 1.0 [28]. The fecal characteristic scores were calculated based on the scores selected by the participants from five optional scores (1-5) against each characteristic in reference to the Bristol stool form scale [29]. The five optional scores were as follows: fecal shape, 1 (very hard), 2 (hard), 3 (smooth), 4 (soft), and 5 (watery); fecal color, 1 (black), 2 (dark brown), 3 (brown), 4 (ocher), and 5 (yellow); fecal odor, 1 (very strong), 2 (strong), 3 (moderate), 4 (almost no odor), and 5 (odorless); and sensation after defecation, 1 (very unrefreshing), 2 (unrefreshing), 3 (general), 4 (refreshing), and 5 (very refreshing).

\subsection{Fecal $\mathrm{pH}$ and SCFA Concentration}

Measurements of fecal $\mathrm{pH}$ and SCFA concentration were performed at the TechnoSuruga Laboratory Co., Ltd. After sampling, the $\mathrm{pH}$ was measured immediately using a digital $\mathrm{pH}$ meter (LAQUAtwin B-712, HORIBA, Ltd., Kyoto, Japan). For measuring the organic acid content, $0.1 \mathrm{~g}$ of each fecal sample was placed in a $2.0 \mathrm{~mL}$ tube with zirconia beads and suspended in Milli-Q ultrapure water. The samples were heated at $85{ }^{\circ} \mathrm{C}$ for $15 \mathrm{~min}$, vortexed at $5 \mathrm{~m} / \mathrm{s}$ for $45 \mathrm{~s}$ using FastPrep-24 (MP Biomedicals, Santa Ana, CA, USA), and centrifuged at $15,350 \times g$ for $10 \mathrm{~min}$. The supernatant was filtered using a $0.2 \mu \mathrm{m}$ filter (Ultrafree-MC PTFE LG; Merck Millipore, Danvers, MA, USA) and analyzed for the detection of nine fatty acids: succinic acid, lactic acid, and SCFAs (formic acid, acetic acid, propionic acid, iso-butyric acid, $n$-butyric acid, iso-valeric acid, and $n$-valeric acid) using an organic acid analysis system (Shimadzu Corp., Kyoto, Japan) with a Prominence ${ }^{\mathrm{TM}}$ high-performance liquid chromatography system, a conductivity detector (CDD-10A (Shimadzu)), two columns arranged in tandem (Shim-pack SCR-102(H) (300 mm $\times 8 \mathrm{~mm} \mathrm{ID)),}$ a guard column (Shim-pack SCR-102(H) $(50 \mathrm{~mm} \times 6 \mathrm{~mm} \mathrm{ID)})$, a mobile phase $(5 \mathrm{mM}$ $p$-toluenesulfonic acid), and a reaction solution containing $5 \mathrm{mM} p$-toluenesulfonic acid, $100 \mu \mathrm{M}$ ethylenediaminetetraacetic acid, and $20 \mathrm{mM}$ bis(2-hydroxyethyl)iminotris (hydroxymethyl)methane. The flow rate and oven temperature were $0.8 \mathrm{~mL} / \mathrm{min}$ and $45^{\circ} \mathrm{C}$, respectively. The limits of quantification were $0.05 \mathrm{mg} / \mathrm{g}$ for succinic acid, lactic acid, 
acetic acid, and propionic acid and $0.10 \mathrm{mg} / \mathrm{g}$ for formic acid, iso-butyric acid, $n$-butyric acid, iso-valeric acid, and $n$-valeric acid, respectively.

\subsection{DNA Extraction and Next-Generation Sequencing (NGS) Analysis of Bacterial Flora in Feces}

Genomic DNA was extracted from the fecal samples, and NGS analysis was performed at the TechnoSuruga Laboratory Co., Ltd. Fecal solids in the suspension were ground with zirconia beads using a FastPrep-24 Instrument (MP Biomedicals) at $5 \mathrm{~m} / \mathrm{s}$ for $2 \mathrm{~min}$. Bacterial DNA was extracted from $200 \mu \mathrm{L}$ of the suspension using an automated DNA extraction machine (GENE PREP STAR PI-480, Kurabo Industries Ltd., Osaka, Japan) according to the manufacturer's instructions. The DNA content measured using a NanoDrop ${ }^{\mathrm{TM}}$ ND-8000 (NanoDrop Technologies, Wilmington, DE, USA), and the final concentration (10 ng/ $\mu \mathrm{L})$ of the DNA sample was noted.

NGS analysis of bacterial 16S rRNA gene (16S rDNA) was performed using MiSeq (Illumina, San Diego, CA, USA), as previously reported by Takahashi et al. [30]. Briefly, the V3-V4 hypervariable regions of $16 \mathrm{~S}$ rDNA were amplified using 341F and 806R, the universal primers for bacteria, and touchdown PCR was performed using a GeneAmp PCR system 9700 (Applied Biosystems, Foster City, CA, USA) [30,31]. Sequencing was performed using a paired-end method and modified to a $2 \times 300$ bp cycle run using an Illumina MiSeq sequencing system (Illumina, San Diego, CA, USA) and MiSeq Reagent Kit version 3 (600 cycle) chemistry. Paired-end sequencing was performed with a read length of $301 \mathrm{bp}$ [32]. After demultiplexing, a clear overlap was observed in the paired-end reads. The sequences were subjected to quality checks and filtering using the Fast $X$ toolkit [33], and only reads having $>99 \%$ of their sequence with quality value scores $\geq 20$ were extracted for further analysis. The chimeric sequences were eliminated using the USEARCH software $[34,35]$. Based on the sequences, the taxonomic positions were identified at $97 \%$ similarity using the Metagenome@KIN analysis software (World Fusion, Tokyo) and the TechnoSuruga Lab Microbial Identification database (DB-BA 13.0; TechnoSuruga Laboratory), which only lists bacteria with standing in the taxonomic nomenclature $[31,36,37]$.

\subsection{Statistical Analysis}

Results are presented in terms of the mean values and standard deviations. Intergroup comparisons between the koji amazake and placebo groups were performed using repeated measures analysis of variance (ANOVA), followed by an unpaired $t$-test. Intragroup differences in the values at weeks 3 and 5 vs. those at week 0 (baseline) were compared using Dunnett's multiple comparisons test. Values with $p<0.05$ were considered statistically significant, and those with $p<0.1$ were considered to be trending toward significance. Statistical analyses were performed using the EZR software (version 1.27; Saitama Medical Center, Jichi Medical University, Saitama, Japan).

\section{Results}

\subsection{Analysis of Functional Components Derived from A. oryzae}

To reveal functional components in koji amazake manufactured by $A$. oryzae-derived fermentation process, we intensively investigated contents of oligosaccharides, GlcCer, and A. oryzae cells as its candidates. Furthermore, these candidates are reported to have the ability to modify the intestinal microbiome $[18,22,23]$. The nutrient composition of the test beverages (koji amazake and placebo) is shown in Table 1 . The beverages only had minor differences with respect to the nutritional values of proteins, fats, and carbohydrates. In fact, the prebiotic oligosaccharide content in koji amazake (3.12 $\pm 0.03 \mathrm{~g})$ was only 1.26 times greater than that in the placebo $(2.47 \pm 0.07 \mathrm{~g})$. Although raffinose and gentiobiose have been reported to be contained in koji amazake [13], these oligosaccharides were not detected in the present study. Some prebiotic oligosaccharides, such as isomaltose and kojibiose, were produced by the transglycosylation of $\alpha$-glucosidase. In particular, panose and kojibiose was contained at only low level, but these oligosaccharides were specifically produced by A. oryzae. Conversely, the oligosaccharides present in the saccharified solution 
were primarily IMOs derived from the branched chain of amylopectin. The GlcCer was localized on plasma membrane and plays essential physiological roles in fungi [22]. A. oryzae-derived GlcCer have unique $\mathrm{C}_{9}$-metylated spingoid base that was not contained in rice [19]. koji amazake had a GlcCer content of $1.39 \pm 0.12 \mathrm{mg}$, which was 3.4 times greater than that in the placebo $(0.41 \pm 0.04 \mathrm{mg})$. The GlcCer content comprised two types of A. oryzae-derived GlcCer (Figure S1A,B) and six types of rice-derived GlcCer (Figure $\mathrm{S} 1 \mathrm{C}-\mathrm{H})$. The chemical structures of GlcCer have also been reported earlier $[21,38,39]$. A. oryzae-derived GlcCer was not present in the placebo. The galactosylceramide was not detected in the beverages. Main materials for koji amazake are rice-koji which is steamed rice fermented by $A$. oryzae. Therefore, $A$. oryzae cells ( $302 \pm 15.5 \mathrm{mg}$ ) were detected in koji amazake. Conversely, A. oryzae cells were not detected in placebo due to the enzymatic manufacturing process.

\subsection{Participants and Their Background Characteristics}

All 44 participants (18 males and 26 females) successfully completed this trial without any drop-outs, and data from all participants were included in the final analysis. The results of the hematological and biochemical tests conducted at the screening visit were within the normal range, and the representative background characteristics of the 22 participants in each group are summarized in Table S1. The weekly defecation frequency values were $3.95 \pm 1.02$ days/week and $4.68 \pm 1.55$ times/week in the koji amazake group and $4.00 \pm 1.04$ days/week and $4.73 \pm 1.63$ times/week in the placebo group. There were no significant differences ( $p=0.773$ and 0.927 , respectively) in the background characteristics between the two groups, as determined using an unpaired $t$-test. No adverse events were reported during the 6-week study period.

\subsection{Defecation Patterns and Fecal Characteristics}

Based on the data recorded in the questionnaires, the weekly values of defecation patterns (frequency and weight) and fecal characteristics of participants from the koji amazake and placebo groups during the 6-week study period are summarized in Table 2, along with the results of the statistical analysis of inter- and intra-group comparisons of the two groups. The number of weekly defecation days, defecation frequency, and fecal weight increased significantly in the koji amazake group compared with the placebo group, as evaluated using repeated measures ANOVA followed by an unpaired $t$-test at each time point. Weekly defecation days and frequency of koji amazake group increased significantly at $2 \mathrm{w}(p=0.037$ and 0.004$)$, at $3 \mathrm{w}(p=0.010$ and 0.035$)$, and at $4 \mathrm{w}(p=0.027$ and 0.009$)$, compared with the corresponding values of the placebo group. Weekly fecal weight of the koji amazake group increased significantly at $4 \mathrm{w}(p=0.028)$ and showed a trend to increase at $2 \mathrm{w}(p=0.063)$. In terms of changes $(\Delta)$ from baseline $(0 \mathrm{w})$ till the five time points, the $\Delta$ defecation frequency (number of days), $\Delta$ defecation frequency (number of times), and $\Delta$ fecal weight also increased significantly in the koji amazake group compared with the placebo group. The values obtained were as follows: weekly $\Delta$ frequency (number of days) at $2 \mathrm{w}(1.14 \pm 1.39$ days vs. $0.14 \pm 1.36$ days, $p=0.020), 3 \mathrm{w}(1.45 \pm 1.34$ days vs. $0.18 \pm 1.40$ days, $p=0.004)$, and $4 \mathrm{w}(1.14 \pm 1.58$ days vs. $0.08 \pm 1.43$ days, $p=0.013)$; weekly $\Delta$ frequency (number of times) at $2 \mathrm{w}(1.64 \pm 1.92$ times vs. $-0.23 \pm 1.92$ times, $p=0.003), 3 \mathrm{w}(2.05 \pm 2.63$ times vs. $0.36 \pm 1.97$ times, $p=0.021)$, and $4 \mathrm{w}(1.64 \pm 2.36$ times vs. $-0.23 \pm 1.90$ times, $p=0.006)$; and weekly $\Delta$ fecal weight at $2 \mathrm{w}(194 \pm 305 \mathrm{~g}$ vs. $34 \pm 214 \mathrm{~g}, p=0.052$ (a trend)) and $4 \mathrm{w}(206 \pm 358 \mathrm{~g}$ vs. $17 \pm 268 \mathrm{~g}, p=0.054$ (a trend)) (Figure 1). 
Table 2. Weekly values and the statistical analysis of defecation patterns (frequency and weight) and fecal characteristics of a koji amazake group and placebo groups during a 6-week study period.

\begin{tabular}{|c|c|c|c|c|c|c|c|c|}
\hline \multirow[b]{2}{*}{ Group $n$} & \multirow{2}{*}{$\begin{array}{l}\text { Time Point } \\
\text { (Period) }^{(1)}\end{array}$} & \multicolumn{3}{|c|}{ Weekly Defecation Patterns ${ }^{(3,4)}$} & \multicolumn{4}{|c|}{ Scores of Fecal Characteristics $(2,3,4)$} \\
\hline & & $\begin{array}{l}\text { Defecation Days } \\
\text { (Days/Week) }\end{array}$ & $\begin{array}{c}\text { Frequency } \\
\text { (Times/Week) }\end{array}$ & $\begin{array}{c}\text { Weight } \\
\text { (g/Week) }\end{array}$ & Shape & Color & Odor & Sensation \\
\hline \multirow{6}{*}{$\begin{array}{c}\text { koji amazake } \\
n=22 \\
\text { age range } \\
20-66\end{array}$} & $0 \mathrm{w}(-1$ to $0 \mathrm{w})$ & $3.95 \pm 1.05^{\mathrm{a}}$ & $4.68 \pm 1.59^{a}$ & $518 \pm 277$ & $3.24 \pm 0.49$ & $2.76 \pm 0.40^{a}$ & $2.89 \pm 0.67$ & $3.31 \pm 0.88$ \\
\hline & $1 \mathrm{w}(0$ to $1 \mathrm{w})$ & $4.73 \pm 1.75^{\mathrm{a}}$ & $5.95 \pm 2.61^{\mathrm{a}}$ & $616 \pm 330$ & $3.08 \pm 0.51$ & $3.01 \pm 0.39^{a}$ & $2.96 \pm 0.38$ & $3.15 \pm 0.78$ \\
\hline & $2 \mathrm{w}(1$ to $2 \mathrm{w})$ & $5.09 \pm 1.51^{\mathrm{a}, *}$ & $6.32 \pm 2.21^{a, *}$ & $712 \pm 385^{\#}$ & $3.02 \pm 0.44$ & $3.11 \pm 0.38^{b}$ & $2.96 \pm 0.43$ & $3.42 \pm 0.84$ \\
\hline & $3 w(2$ to $3 w)$ & $5.41 \pm 1.53^{b, *}$ & $6.73 \pm 2.76^{b, * *}$ & $752 \pm 360$ & $2.97 \pm 0.40$ & $3.21 \pm 0.49^{b}$ & $2.88 \pm 0.42$ & $3.31 \pm 0.65$ \\
\hline & $4 \mathrm{w}(3$ to $4 \mathrm{w})$ & $5.09 \pm 1.80^{\mathrm{a}, *}$ & $6.32 \pm 2.53^{a, * *}$ & $724 \pm 402 *$ & $3.11 \pm 0.36$ & $2.97 \pm 0.45^{\mathrm{a}}$ & $2.78 \pm 0.45$ & $3.20 \pm 0.78$ \\
\hline & $5 \mathrm{w}(4$ to $5 \mathrm{w})$ & $4.86 \pm 1.55^{\mathrm{a}}$ & $6.00 \pm 2.60^{a}$ & $665 \pm 387$ & $3.11 \pm 0.32$ & $3.03 \pm 0.41^{\mathrm{a}}$ & $2.82 \pm 0.38$ & $3.43 \pm 0.70$ \\
\hline \multirow{6}{*}{$\begin{array}{c}\text { Placebo } \\
n=22 \\
\text { age range } \\
22-66\end{array}$} & $0 \mathrm{w}(-1$ to $0 \mathrm{w})$ & $4.00 \pm 1.07$ & $4.73 \pm 1.67$ & $484 \pm 235$ & $3.34 \pm 0.58$ & $2.78 \pm 0.54$ & $2.86 \pm 0.72$ & $3.02 \pm 0.85$ \\
\hline & $1 \mathrm{w}(0$ to $1 \mathrm{w})$ & $4.00 \pm 1.41$ & $4.95 \pm 2.03$ & $515 \pm 264$ & $3.16 \pm 0.54$ & $3.04 \pm 0.54$ & $3.15 \pm 0.54$ & $3.17 \pm 0.60$ \\
\hline & $2 \mathrm{w}(1$ to $3 \mathrm{w})$ & $4.14 \pm 1.42$ & $4.50 \pm 1.71$ & $519 \pm 272$ & $3.15 \pm 0.67$ & $3.08 \pm 0.60$ & $3.01 \pm 0.60$ & $3.40 \pm 0.71$ \\
\hline & $3 \mathrm{w}(2$ to $3 w)$ & $4.18 \pm 1.50$ & $5.09 \pm 9.18$ & $647 \pm 386$ & $3.27 \pm 0.70$ & $3.06 \pm 0.54$ & $2.92 \pm 0.56$ & $3.15 \pm 0.70$ \\
\hline & $4 \mathrm{w}(3$ to $4 \mathrm{w})$ & $3.95 \pm 1.46$ & $4.50 \pm 1.85$ & $501 \pm 225$ & $3.31 \pm 0.66$ & $3.07 \pm 0.51$ & $2.86 \pm 0.58$ & $3.12 \pm 0.73$ \\
\hline & $5 \mathrm{w}(4$ to $5 \mathrm{w})$ & $4.77 \pm 1.34$ & $5.59 \pm 1.94$ & $681 \pm 441$ & $3.45 \pm 0.64$ & $3.04 \pm 0.50$ & $3.00 \pm 0.50$ & $3.26 \pm 0.64$ \\
\hline \multirow{3}{*}{$\begin{array}{l}\text { ANOVA } \\
p \text { value }^{(5)}\end{array}$} & Group & 0.0638 & 0.0463 & 0.2296 & 0.0886 & 0.9871 & 0.5117 & 0.4944 \\
\hline & Time & 0.0003 & 0.0011 & 0.0000 & 0.1035 & 0.0001 & 0.0350 & 0.1135 \\
\hline & Group $\times$ time & 0.0036 & 0.0031 & 0.0282 & 0.6584 & 0.7229 & 0.6205 & 0.8051 \\
\hline
\end{tabular}

The values are the mean \pm standard deviation. (1) Each time point was the last day of each period; a non-intake observation period ( -1 to $0 \mathrm{w}$ ), intake period ( 0 to $1 \mathrm{w}, 1$ to $2 \mathrm{w}$, and ( 2 to $3 \mathrm{w}$ ), and follow-up period ( 3 to $4 \mathrm{w}$ and 4 to $5 \mathrm{w}$ ). (2) Scores were calculated based on the scores selected from 5 optional scores described in Materials and Methods. ${ }^{(3)} * *, *$, \# Values within a column with asterisk indicate significant difference $(* * p<0.01, * p<0.05)$ and trends towards significance $(\# p<0.1)$ with inter-group comparison between the corresponding time points by two-way repeated measures analysis of variance (ANOVA) followed by unpaired $t$-test. ${ }^{(4)} \mathrm{a}$, b Values within a column with different superscript alphabets indicate significant difference $(p<0.05)$ with intra-group comparison among six time points by Dunnett's multiple comparison test. ${ }^{(5)}$ By repeated measures analysis of variance (ANOVA): group (between-subjects effect), time (within-subjects effects), group $\times$ time (interactions).

A
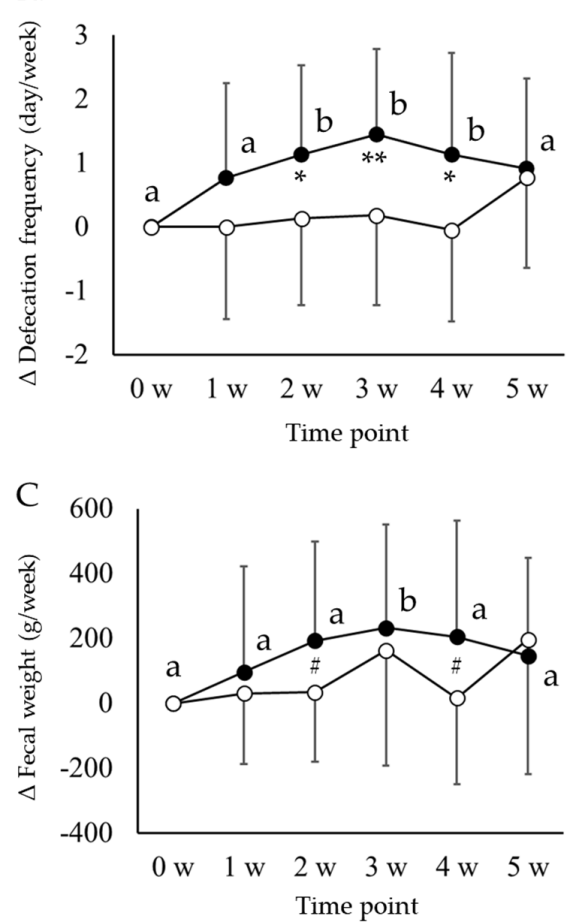

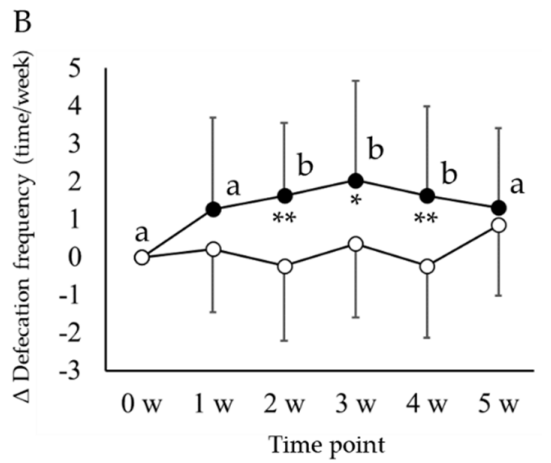

Figure 1. Comparison of the changes $(\Delta)$ in weekly defecation frequencies ((A) days per week and (B) times per week) and fecal weight $((\mathbf{C}) \mathrm{g}$ per week) from baseline values $(0 \mathrm{w})$ in the koji amazake group (filled circle) and placebo group (empty circle). Each value is expressed as mean \pm standard deviation. Inter-group comparisons between the koji amazake and placebo groups were performed using repeated measures analysis of variance (ANOVA) followed by a paired $t$-test; significant differences are indicated by ${ }^{* *} p<0.01,{ }^{*} p<0.05$, and $\# p<0.1$. a, b Intra-group changes from baseline values $(0 \mathrm{w})$ in each group at six time points were compared using Dunnett's multiple comparisons test; values with different superscript letters in each profile indicate significant difference $(p<0.05)$. 
To evaluate the effects of koji amazake during intake and after termination of intake, the intra-group changes from baseline $(0 \mathrm{w})$ to 3 and $5 \mathrm{w}$ were compared using Dunnett's multiple comparisons test. The values in the koji amazake group increased significantly (presented as items (time point, value vs. value at $0 \mathrm{w} ; p$ value)): weekly frequency (number of days) ( $3 \mathrm{w}, 5.41 \pm 1.53$ days vs. $3.95 \pm 1.05$ days; $p=0.025$ ), weekly frequency (number of times) ( $3 \mathrm{w}, 6.73 \pm 2.76$ times vs. $4.68 \pm 1.59$ times; $p=0.025)$, and color $(2 \mathrm{w}, 3.11 \pm 0.38$ and $3 \mathrm{w}, 3.21 \pm 0.49$ vs. $2.76 \pm 0.40 ; p=0.032$ and 0.003 , respectively). However, the significant differences observed initially were not observed after 5 weeks of follow-up. The placebo group showed no significant intra-group differences in defecation patterns or fecal characteristic scores at 3 or $5 \mathrm{w}$.

\subsection{Fecal $p H$ and SCFA Concentration}

The fecal $\mathrm{pH}$ and fatty acid concentration in the koji amazake and placebo group were measured on the last day of each study period during the non-intake observation period $(0 \mathrm{w})$, intake period $(3 \mathrm{w})$, and follow-up period $(5 \mathrm{w})$, and the following $\mathrm{pH}$ values were obtained: koji amazake group, $7.02 \pm 0.49,6.97 \pm 0.64$, and $7.09 \pm 0.43$; placebo group, $7.19 \pm 0.57,7.00 \pm 0.37$, and $7.1 \pm 0.42$, respectively. The $\mathrm{pH}$ values of fecal samples from the koji amazake and placebo groups at $3 \mathrm{w}$ were lower than those at baseline $(0 \mathrm{w})$ or $5 \mathrm{w}$; however, no statistically significant difference was observed in either inter-group or intra-group comparisons. At each time point, the fecal concentrations of succinic acid, lactic acid, formic acid, acetic acid, propionic acid, iso-butyric acid, $n$-butyric acid, iso-valeric acid, and $n$-valeric acid were measured using gas chromatography mass spectrometry (GC-MS), and the detection rates of these fatty acids in the participants throughout the study period were $72.7 \%, 11.4 \%, 1.5 \%, 100.0 \%, 100.0 \%, 53.8 \%, 99.2 \%, 75.0 \%$, and $66.7 \%$, respectively. Furthermore, the weekly value for the fecal fatty acid weight was calculated by multiplying the concentration with the corresponding weekly fecal weight. The results are shown in Table 3; the results for lactic acid and formic acid were not provided owing to the low detection rates $(<20 \%)$. No significant intergroup difference was observed in either concentration or weekly weight of each fatty acid at any time point. In the intra-group comparison, the total SCFA concentration increased significantly from baseline $(0 \mathrm{w})$ to $3 \mathrm{w}$ in the placebo group, but the increase in the koji amazake group was not significant. However, the weekly weights of total SCFA and total fatty acids increased significantly from baseline $(0 \mathrm{w})$ to $3 \mathrm{w}$ in both groups.

Table 3. Fecal fatty acid concentrations and weekly fecal fatty acid weights of koji amazake and placebo groups on the last day of non-intake observation, intake, and follow up periods. ${ }^{(1,2)}$.

\begin{tabular}{|c|c|c|c|c|c|c|c|}
\hline \multirow{2}{*}{ Fatty Acid } & \multirow{2}{*}{ Group } & \multicolumn{3}{|c|}{ Fecal Fatty Acid Concentration, $\mu \mathrm{mol} / \mathrm{g}$ Feces } & \multicolumn{3}{|c|}{ Weekly Fecal Fatty Acid Weight, mmol } \\
\hline & & $0 \mathbf{w}$ & $3 \mathbf{w}$ & $5 \mathrm{w}$ & $\mathbf{0} \mathbf{w}$ & $3 \mathbf{w}$ & $5 \mathrm{w}$ \\
\hline \multirow{2}{*}{$\begin{array}{l}\text { Succinic } \\
\text { acid }\end{array}$} & amazake & $2.22 \pm 5.44$ & $1.98 \pm 4.36$ & $5.02 \pm 9.74$ & $0.56 \pm 0.98$ & $1.09 \pm 2.51$ & $1.44 \pm 2.91$ \\
\hline & Placebo & $1.33 \pm 0.92$ & $2.86 \pm 4.93$ & $3.13 \pm 6.16$ & $0.52 \pm 0.89$ & $2.51 \pm 3.02$ & $1.73 \pm 3.95$ \\
\hline \multirow{2}{*}{$\begin{array}{c}\text { Acetic } \\
\text { acid }\end{array}$} & amazake & $41.3 \pm 15.1$ & $48.1 \pm 17.2$ & $47.0 \pm 12.7$ & $21.7 \pm 15.3^{a}$ & $37.0 \pm 27.3^{b}$ & $32.1 \pm 23.4^{\mathrm{a}}$ \\
\hline & Placebo & $31.3 \pm 15.8^{\mathrm{a}}$ & $44.8 \pm 16.4^{\mathrm{b}}$ & $42.6 \pm 19.4^{\mathrm{a}}$ & $16.7 \pm 14.8$ & $30.3 \pm 27.9$ & $26.9 \pm 19.7$ \\
\hline \multirow{2}{*}{$\begin{array}{l}\text { Propionic } \\
\text { acid }\end{array}$} & amazake & $13.0 \pm 5.5$ & $15.9 \pm 8.5$ & $13.6 \pm 5.5$ & $7.29 \pm 6.26$ & $12.87 \pm 11.08$ & $9.18 \pm 7.68$ \\
\hline & Placebo & $10.8 \pm 4.1^{\mathrm{a}}$ & $15.8 \pm 7.0^{b}$ & $14.6 \pm 7.5^{\mathrm{a}}$ & $5.25 \pm 3.37^{a}$ & $11.08 \pm 10.65^{b}$ & $9.13 \pm 6.40^{a}$ \\
\hline \multirow{2}{*}{$\begin{array}{l}\text { iso-Butyric } \\
\text { acid }\end{array}$} & amazake & $2.03 \pm 0.92$ & $1.37 \pm 0.67$ & $1.77 \pm 0.85$ & $0.37 \pm 0.79$ & $0.79 \pm 0.84$ & $0.65 \pm 0.90$ \\
\hline & Placebo & $1.59 \pm 0.59$ & $1.80 \pm 0.54$ & $2.04 \pm 0.77$ & $0.28 \pm 0.36^{\mathrm{a}}$ & $0.84 \pm 0.83^{b}$ & $0.53 \pm 0.68^{a}$ \\
\hline \multirow{2}{*}{$\begin{array}{c}n \text {-Butyric } \\
\text { acid }\end{array}$} & amazake & $8.95 \pm 5.13$ & $10.18 \pm 5.97$ & $9.99 \pm 5.88$ & $4.42 \pm 2.92^{a}$ & $7.83 \pm 6.72^{b}$ & $6.71 \pm 6.49^{a}$ \\
\hline & Placebo & $6.98 \pm 4.12$ & $9.27 \pm 4.71$ & $10.05 \pm 7.51$ & $3.69 \pm 3.88$ & $6.72 \pm 4.51$ & $6.49 \pm 6.22$ \\
\hline \multirow{2}{*}{$\begin{array}{l}\text { iso-Valeric } \\
\text { acid }\end{array}$} & amazake & $2.68 \pm 1.50$ & $2.08 \pm 0.79$ & $2.18 \pm 1.44$ & $0.79 \pm 1.31$ & $1.35 \pm 1.07$ & $1.15 \pm 1.30$ \\
\hline & Placebo & $2.18 \pm 1.06$ & $2.48 \pm 1.15$ & $2.39 \pm 1.21$ & $0.67 \pm 0.52$ & $1.07 \pm 1.07$ & $1.28 \pm 1.07$ \\
\hline \multirow{2}{*}{$\begin{array}{l}n \text {-Valeric } \\
\text { acid }\end{array}$} & amazake & $2.71 \pm 2.57$ & $2.18 \pm 0.84$ & $1.91 \pm 0.82$ & $0.78 \pm 1.19$ & $1.36 \pm 1.26$ & $1.11 \pm 1.21$ \\
\hline & Placebo & $1.38 \pm 0.39$ & $2.08 \pm 1.05$ & $2.14 \pm 0.93$ & $0.35 \pm 0.38^{a}$ & $1.26 \pm 1.06^{b}$ & $0.73 \pm 0.83^{a}$ \\
\hline
\end{tabular}


Table 3. Cont.

\begin{tabular}{|c|c|c|c|c|c|c|c|}
\hline \multirow{2}{*}{ Fatty Acid } & \multirow{2}{*}{ Group } & \multicolumn{3}{|c|}{ Fecal Fatty Acid Concentration, $\mu \mathrm{mol} / \mathrm{g}$ Feces } & \multicolumn{3}{|c|}{ Weekly Fecal Fatty Acid Weight, mmol } \\
\hline & & $0 \mathbf{w}$ & $3 \mathrm{w}$ & 5 w & $0 \mathbf{w}$ & $3 \mathbf{w}$ & $5 \mathrm{w}$ \\
\hline Total & amazake & $66.8 \pm 24.8$ & $78.4 \pm 29.9$ & $74.8 \pm 18.9$ & $35.3 \pm 24.8^{\mathrm{a}}$ & $61.2 \pm 45.6^{b}$ & $50.9 \pm 35.8^{a}$ \\
\hline SCFA & Placebo & $52.2 \pm 21.3^{a}$ & $73.9 \pm 22.6^{b}$ & $71.5 \pm 32.1^{b}$ & $26.9 \pm 21.2^{\mathrm{a}}$ & $41.4 \pm 45.0^{\mathrm{b}}$ & $45.0 \pm 30.3^{a}$ \\
\hline Total fatty & amazake & $74.3 \pm 43.9$ & $85.4 \pm 43.9$ & $78.8 \pm 19.6$ & $38.4 \pm 28.7^{\mathrm{a}}$ & $62.4 \pm 46.4^{\mathrm{b}}$ & $52.4 \pm 35.3^{\mathrm{a}}$ \\
\hline acids & Placebo & $60.7 \pm 27.8^{a}$ & $91.6 \pm 45.3^{b}$ & $74.4 \pm 32.1^{\mathrm{a}}$ & $27.9 \pm 22.9^{\mathrm{a}}$ & $52.7 \pm 44.3^{b}$ & $47.2 \pm 31.6^{\mathrm{a}}$ \\
\hline
\end{tabular}

The values are the mean \pm standard deviation, $n=22 .{ }^{(1)}$ Feces were collected on the last day of the following periods; non-intake observation period ( -1 to $0 \mathrm{w}$ ), intake period ( 0 to $3 \mathrm{w}$ ), and follow-up period ( 3 to $5 \mathrm{w})$. (2) a, b Values with different superscript alphabets within a row of each measuring item indicate significant difference $(p<0.05)$ with intra-group comparison with corresponding baseline $(0 \mathrm{w})$ values by Dunnett's multiple comparison test.

\subsection{NGS Analysis of Bacterial Flora in Feces}

A total of 4,879,397 filtered high-quality sequence reads were generated, including the following (results are presented as time point, total reads of 44 samples, average \pm SD per sample, max, and min): $0 \mathrm{w}, 672,457,15,283 \pm 3890,24,479,10,338 ; 3 \mathrm{w}, 2034.436$, $46,237 \pm 9.036,77,124,31,283 ; 5$ w, 2,172,504, 49,375 $\pm 6759,74,864,34,688$. Eight bacterial phyla were identified (Table S2). The dominant phyla were Firmicutes, Actinobacteria, Bacteroidetes, Verrucomicrobia, and Proteobacteria, and members of these five phyla constituted an average of 97.8\% (range 97.3-97.8\%) in both groups. As shown in Figure 2A, statistical analysis of the relative abundance of each phylum revealed no significant differences in the inter-group and intra-group comparisons. The Firmicutes-to-Bacteroidetes ratios of koji amazake and the placebo group were $26.7 \pm 33.4$ and $7.5 \pm 4.0$ at $0 \mathrm{w}, 15.8 \pm 28.3$ and $32.5 \pm 83.2$ at $3 \mathrm{w}$, and $20.7 \pm 30.7$ and $48.1 \pm 170.3$ at $5 \mathrm{w}$, respectively. The difference of mean values with large standard deviations showed the ratios of participants in both two groups were spread out over a large range even at $0 \mathrm{w}$, and there was no inter- and intra-group significant difference. However, analysis of the inter-group comparison of the changes $(\Delta)$ in relative abundance from baseline $(0 \mathrm{w})$ to 3 and $5 \mathrm{w}$ showed significant differences in $\Delta$ Firmicutes (relative abundance) $(-8.51 \%$ vs. $-0.43 \%, p=0.0212)$ and $\Delta$ Bacteroidetes $(3.56 \%$ vs. $2.44 \%, p=0.0167)$ at $3 \mathrm{w}$. Furthermore, the $\Delta$ Firmicutes value decreased significantly from baseline $(0 \mathrm{w})$ to $3 \mathrm{w}(p=0.0033)$ in the intra-group comparison of the koji amazake group (Figure 2B).

A

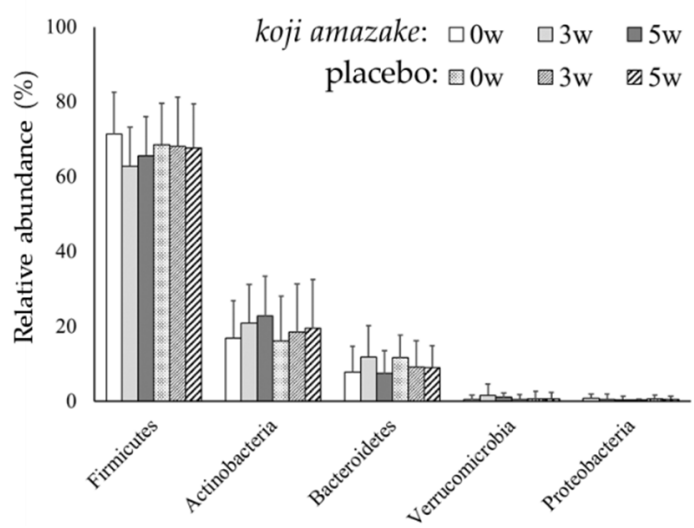

$\mathrm{B}$

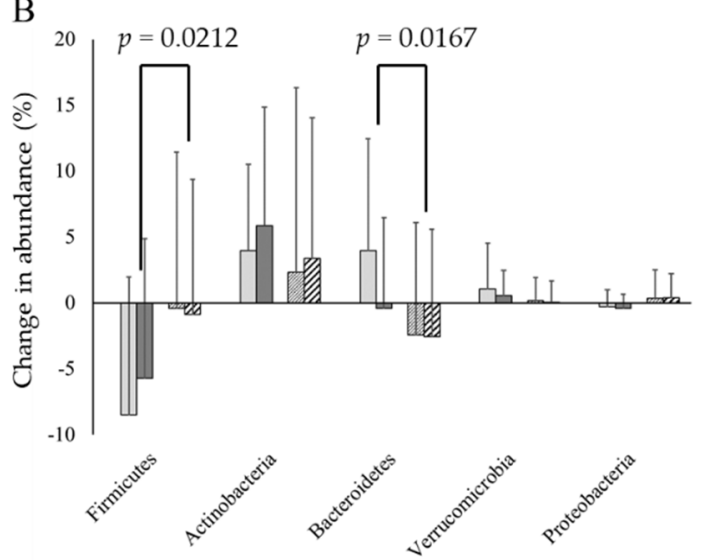

Figure 2. Inter - and intra-group comparison of relative abundances (A) at three time points (0, 3, and $5 \mathrm{w})$, and changes in the abundances of five dominant bacterial phyla (B) from baseline values $(0 \mathrm{w})$ in the koji amazake and placebo groups. Each value is expressed as mean \pm standard deviation. The $p$ values indicate significant inter-group difference, analyzed using repeated measures ANOVA (Firmicutes: group $p=0.0146$, time $p=0.0103$, group $\times$ time $p=0.0282$; Bacteroidetes: group $p=0.0516$, time $p=0.164$, group $\times$ time $p=0.0250$ ) followed by a $t$-test. a, b Columns with different letters indicate significant difference $(p<0.05)$, with intra-group comparisons among three time points in each group of each phylum using Dunnett's multiple comparisons test. 
There were 229 genera with relative abundances more than $0.01 \%$ and $19-21$ genera $(8.3 \%-9.2 \%$ of all detected genera) with relative abundances more than $1.0 \%$, depending on the time point of measurement and group. Table 4 shows the comparison of relative abundances of taxa in the koji amazake and placebo groups at the three time points $(0$, 3 , and $5 \mathrm{w}$ ), with each bacterial genus showing a relative abundance greater than $1.0 \%$. No significant difference was observed in the inter-group comparison (using repeated measures ANOVA followed by $t$-test) or intra-group comparison (using Dunnett's multiple comparisons test) of each genus. However, the inter-group comparison of the change $(\Delta)$ in relative abundance from baseline $(0 \mathrm{w})$ to 3 and $5 \mathrm{w}$ showed significant differences, as shown in Figure 3. In the koji amazake group, a significant decrease was observed for $\Delta$ Blautia ( $3 \mathrm{w},-2.37 \%$ vs. $2.25 \%, p=0.0075)$, and a significant increase was observed for $\Delta$ Bacteroides ( $3 \mathrm{w}, 2.40 \%$ vs. $2.38 \%, p=0.0337$ ). Furthermore, the intra-group comparison revealed a significant increase in $\Delta$ Collinsella at $3 \mathrm{w}$ both in the koji amazake and placebo groups, as determined using Dunnett's multiple comparisons test.

Table 4. Comparison of relative abundances at three time points $(0 \mathrm{w}, 3 \mathrm{w}$, and $5 \mathrm{w})$ of koji amazake and placebo groups in each bacterial genus with relative abundance more than $1.0 \%$.

\begin{tabular}{|c|c|c|c|c|c|c|}
\hline \multirow{2}{*}{ Genera } & \multicolumn{3}{|c|}{ Relative Abundance $\%$, Koji Amazake $n=22$} & \multicolumn{3}{|c|}{ Relative Abundance $\%$, Placebo $n=22$} \\
\hline & $0 \mathbf{w}$ & $3 \mathbf{w}$ & $5 \mathbf{w}$ & $0 \mathbf{w}$ & $3 \mathbf{w}$ & $5 \mathbf{w}$ \\
\hline Blautia & $15.40 \pm 8.62$ & $13.03 \pm 9.65$ & $14.25 \pm 7.64$ & $12.17 \pm 6.45$ & $14.42 \pm 6.07$ & $14.42 \pm 8.71$ \\
\hline Bifidobacterium & $13.57 \pm 8.96$ & $14.91 \pm 8.72$ & $16.66 \pm 9.11$ & $12.38 \pm 10.57$ & $12.31 \pm 11.12$ & $12.31 \pm 12.03$ \\
\hline Bacteroides & $6.56 \pm 5.77$ & $8.96 \pm 6.63$ & $5.94 \pm 5.28$ & $9.52 \pm 5.09$ & $7.14 \pm 6.16$ & $7.14 \pm 5.04$ \\
\hline Lachnospiracea (a) & $6.50 \pm 3.48$ & $7.44 \pm 5.45$ & $6.01 \pm 3.65$ & $6.78 \pm 4.22$ & $6.86 \pm 4.67$ & $6.86 \pm 3.19$ \\
\hline Faecalibacterium & $5.74 \pm 4.60$ & $6.23 \pm 4.13$ & $5.17 \pm 3.39$ & $6.18 \pm 4.72$ & $5.49 \pm 4.50$ & $5.49 \pm 4.60$ \\
\hline Collinsella & $2.84 \pm 2.97$ & $5.01 \pm 4.85$ & $5.26 \pm 4.96$ & $3.15 \pm 2.54$ & $5.58 \pm 4.11$ & $5.58 \pm 3.99$ \\
\hline Gemmiger & $5.36 \pm 4.09$ & $4.42 \pm 3.43$ & $4.83 \pm 4.02$ & $4.95 \pm 5.18$ & $3.36 \pm 2.83$ & $3.36 \pm 5.81$ \\
\hline Ruminococcus & $5.42 \pm 5.72$ & $3.28 \pm 4.01$ & $5.43 \pm 5.64$ & $4.58 \pm 5.63$ & $4.01 \pm 4.53$ & $4.01 \pm 4.86$ \\
\hline Fusicatenibacter & $4.66 \pm 4.10$ & $2.68 \pm 2.40$ & $2.97 \pm 2.97$ & $5.09 \pm 4.13$ & $3.57 \pm 3.05$ & $3.57 \pm 2.83$ \\
\hline Ruminococcus2 & $4.43 \pm 6.23$ & $2.82 \pm 2.70$ & $2.80 \pm 2.48$ & $3.99 \pm 3.17$ & $3.55 \pm 4.01$ & $3.55 \pm 2.47$ \\
\hline Roseburia & $3.31 \pm 3.80$ & $2.31 \pm 2.22$ & $2.46 \pm 1.91$ & $3.00 \pm 3.74$ & $330 \pm 3.86$ & $3.30 \pm 3.85$ \\
\hline Clostridium XlVa & $2.15 \pm 1.65$ & $2.56 \pm 2.11$ & $2.44 \pm 2.49$ & $2.61 \pm 2.38$ & $2.91 \pm 2.35$ & $2.91 \pm 1.64$ \\
\hline Rejected hit & $2.28 \pm 4.90$ & $1.84 \pm 1.87$ & $2.10 \pm 2.98$ & $2.43 \pm 3.25$ & $2.18 \pm 2.84$ & $2.18 \pm 2.26$ \\
\hline Anaerostipes & $3.21 \pm 2.56$ & $2.40 \pm 2.27$ & $2.35 \pm 2.08$ & $2.06 \pm 1.96$ & $1.56 \pm 1.70$ & $1.56 \pm 1.56$ \\
\hline Streptococcus & $1.99 \pm 3.37$ & $1.41 \pm 1.83$ & $1.81 \pm 1.94$ & $1.79 \pm 1.88$ & $2.11 \pm 2.14$ & $2.11 \pm 2.70$ \\
\hline Clostridium IV & $1.32 \pm 2.14$ & $1.02 \pm 1.14$ & $1.49 \pm 1.92$ & $2.85 \pm 3.64$ & $1.68 \pm 2.51$ & $1.68 \pm 3.17$ \\
\hline Clostridium XVIII & $1.30 \pm 1.90$ & $1.41 \pm 1.61$ & $1.24 \pm 1.17$ & $1.13 \pm 1.03$ & $1.63 \pm 1.53$ & $1.63 \pm 1.53$ \\
\hline Dorea & $1.59 \pm 0.95$ & $1.41 \pm 1.73$ & $1.39 \pm 1.33$ & $1.54 \pm 1.76$ & $1.50 \pm 1.39$ & $1.50 \pm 1.34$ \\
\hline Megamonas & $0.04 \pm 0.17$ & $1.40 \pm 4.46$ & $0.43 \pm 1.86$ & $0.62 \pm 2.61$ & $1.49 \pm 6.29$ & $1.49 \pm 8.45$ \\
\hline Megasphaera & $1.05 \pm 2.48$ & $1.37 \pm 2.28$ & $1.49 \pm 2.66$ & $0.38 \pm 1.40$ & $1.59 \pm 6.59$ & $1.59 \pm 2.10$ \\
\hline Prevotella & $0.21 \pm 0.55$ & $1.30 \pm 4.37$ & $0.17 \pm 0.45$ & $0.17 \pm 0.40$ & $0.22 \pm 0.64$ & $0.22 \pm 0.18$ \\
\hline Dialister & $0.88 \pm 1.99$ & $0.98 \pm 1.59$ & $1.35 \pm 2.81$ & $0.61 \pm 0.89$ & $0.97 \pm 1.25$ & $0.86 \pm 1.40$ \\
\hline Clostridium XI & $0.51 \pm 0.74$ & $0.62 \pm 0.69$ & $0.75 \pm 0.99$ & $1.20 \pm 1.04$ & $0.72 \pm 0.68$ & $0.99 \pm 1.40$ \\
\hline Coprococcus & $1.02 \pm 1.56$ & $0.69 \pm 1.00$ & $0.86 \pm 1.26$ & $0.94 \pm 1.08$ & $0.81 \pm 0.99$ & $0.80 \pm 1.12$ \\
\hline
\end{tabular}

(a) Lachnospiracea incertae sedis. The values are the mean \pm standard deviation. No significant difference was observed in either inter-group comparison (by repeated measures ANOVA followed by unpaired $t$-test) or intra-group comparison (by Dunnett's multiple comparison test) of each genus. 


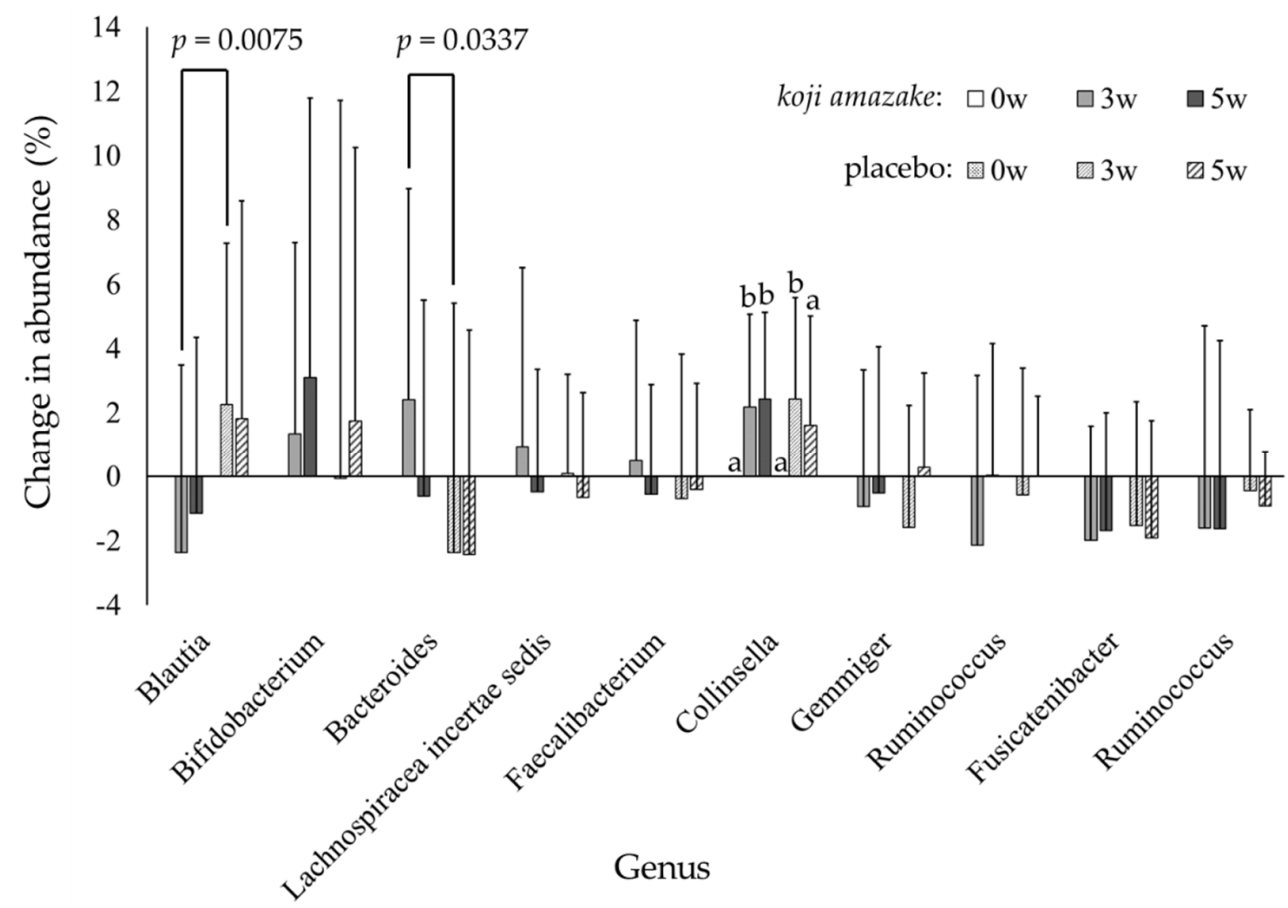

Figure 3. Inter - and intra-group comparison of the changes in $\Delta$ relative abundance from baseline values $(0 \mathrm{w})$ to $3 \mathrm{w}$ and in each of ten dominant bacterial genera of the koji amazake and placebo groups. Each value is expressed as mean \pm standard deviation. Delta $(\Delta)$ relative abundance is the change in relative abundance compared with the value at baseline. Statistical analyses: $p$ values indicate significant inter-group difference, analyzed using repeated measures ANOVA (Blautia: group $p=0.0193$, time $p=0.8846$, group $\times$ time $p=0.0276$; Bacteroidetes: group $p=0.0830$, time $p=0.2191$, group $\times$ time $p=0.0617$ ) followed by a $t$-test. a, b Columns with different letters indicate significant difference $(p<0.05)$, with intra-group comparison in each group of each genus at three time points, as calculated using Dunnett's multiple comparisons test.

\section{Discussion}

The findings of this study showed that the intake of koji amazake including metabolites produced by A. oryzae, a Japanese traditional fermented beverage, was beneficial for increasing the defecation frequency and fecal weight in healthy adults and also for changing the relative abundances of the genera Blautia and Bacteroides in the fecal microbiota.

The increase in the weekly defecation frequency (number of days and number of times) and weekly fecal weight in the koji amazake group was also significant in the intragroup comparison, whereas no significant intra-group changes were noted in the placebo group. However, the significant increase in the values in the koji amazake group for both comparisons was not observable at $5 \mathrm{w}$ after the intake was stopped. With respect to the scores for fecal characteristics, including shape, color, odor, and sensation, no significant differences were observed in the inter-group or intra-group comparisons of the two groups, barring the brightening of the fecal color at 2 and $3 \mathrm{w}$, which resulted in a significant intra-group difference in the koji amazake group.

No statistically significant group difference was observed in fecal $\mathrm{pH}$ and fecal fatty acid concentrations (Table 3). The reasons for this are not completely understood, but we supposed the reason is attributed to the rapid absorption of SCFAs and an increase in fecal weight. SCFAs are the primary energy source for colonocytes and are rapidly absorbed by them [40]. The fecal SCFA concentration was not found to be affected directly by the consumption of prebiotics or probiotics [41,42]. In addition, the fecal SCFA concentration was reportedly affected by fecal characteristics, such as fecal weight and consistency; this was also observed upon the intake of mushrooms, which resulted in a significant increase in stool weight with no significant difference in fecal SCFA concentrations [43]. The rapid absorption of SCFA and an increase in the fecal weight also affected the SCFA 
concentrations. The total SCFA concentration in the koji amazake group was found to increase from baseline $(0 \mathrm{w})$ to $3 \mathrm{w}$, but this increase was not significant. However, the weekly total SCFA weight at $3 \mathrm{w}$ increased significantly compared with that at $0 \mathrm{w}$. These results suggest that some components of koji amazake may function synergistically and contribute to the increase in SCFA.

The 16S rRNA gene profiling revealed that koji amazake intake affected the relative abundances of several dominant taxa of the intestinal microbiota. No significant differences in the relative abundances of either phylum or genus were observed in the inter-group comparisons at each time point between the two groups. However, the inter-group comparison of the $\Delta$ relative abundance from baseline $(0 \mathrm{w})$ to $3 \mathrm{w}$ showed significant changes in dominant taxa. We anticipated an increase in the relative abundance of Bifidobacterium in both groups as compared with corresponding $0 \mathrm{w}$ samples because both beverages contained several kinds of oligosaccharides reported as bifidogenic prebiotics, such as gentiobiose [15], raffinose [16], and isomaltooligosaccharides [17,18]. However, the $\Delta$ Bifidobacterium value did not differ significantly in either inter-group or intra-group comparison. We have previously reported that eight indigestible oligosaccharides are synthesized in koji amazake through A. oryzae fermentation [13]. However, the amounts of oligosaccharides known as prebiotics contained in koji amazake was 1.26-fold relative to that in the placebo (Table 1). The main oligosaccharide was isomaltose-derived branched chains of amylopectin in rice starch. Therefore, isomaltose in koji amazake and placebo were close to $1.96 \mathrm{~g}$ and 1.89 $\mathrm{g}$, respectively. Isomaltooligosaccharaides have been reported to improve defecation frequency in constipated patients with intake of $10 \mathrm{~g} /$ day for 1 week [18]. Since the amount of isomaltose contained in koji amazake is less than that amount, other substances might be involved.

Koji, which is main raw material in koji amazake, contains GlcCer derived from both $A$. oryzae and rice. This study revealed koji amazake contained 3.4-fold GlcCer (1.39 $\pm 0.02 \mathrm{mg})$ relative to the placebo $(0.41 \pm 0.04 \mathrm{mg})$. This significant difference was certainly attributed to A. oryzae (Table 1). The GlcCer derived from plants is known to have an ability to improve skin barrier function at intake of 1.2-1.8 $\mathrm{mg}[44,45]$. An in vitro test has reported that GlcCer extracted from A. oryzae improve skin barrier function through enhancement of the expression of OCLN encoding occluding, which is one of the proteins constituting tight junction [46]. Taken together, GlcCer is a functional substance that works at the mg level; however, further verification is needed to determine whether such a small amount of ceramide contributes to improved bowel movements.

koji amazake also contains $302 \pm 15.5 \mathrm{mg}$ of $A$. oryzae cells. Rodriguez et al. reported that chitin-glucan prepared from the cell wall of $A$. niger induces specific changes intestinal bacteria and further increases the bacterial metabolites in feces, including SCFA [23]. The cell wall of $A$. oryzae is the same as that of $A$. niger, which is composed of chitin, $\alpha-1,3-$ glucan, and $\beta-1,6$-branced $\beta$-1,3-glucan [47]. Therefore, alternation of relative abundance of Blautia and Bacteroides between intake of koji amazake and placebo was probably attributed to the cell wall of $A$. oryzae cells in koji amazake. We conclude that the intestinal microbiome modified by intake of $A$. oryzae cells contained in koji amazake mainly contributed to the improvement in defecation frequency. However, identification in the intestinal microbiome was only feasible up to the genus level. The precise effect of koji amazake on the intestinal microbiome should be investigated in a future study.

Interestingly, the Firmicutes-to-Bacteroidetes ratios at $3 \mathrm{w}$ and $5 \mathrm{w}$ became numerically lower in the koji amazake group than in the placebo group. The Firmicutes-to-Bacteroidetes ratio has been shown to be directly correlated with obesity in several studies, indicating that the ratio increases in individuals who are overweight and obese $[48,49]$. Recently, the Firmicutes-to-Bacteroidetes ratio was reported to decrease based on dietary intake, such as the intake of soy milk [50] or probiotics [51]. Manipulation of the ratio based on the alteration of dietary components is expected to serve as a potential therapeutic approach for the prevention and management of obesity. Therefore, koji amazake might be one of the dietary candidates with the potential to decrease the Firmicutes-to-Bacteroidetes ratio. 
Several mechanisms have been reported to explain the improvement in defecation frequency following the intake of probiotics and prebiotics, such as enhancing colonic peristalsis by SCFA generation, increasing stool bulking by alteration of microbial composition, and promoting softness of feces [10]. The results of the present study strongly suggest that an increase in stool bulking by the alteration of the intestinal microbiota may improve the defecation frequency.

In conclusion, the results of this study reveal that the intake of koji amazake improved defecation by increasing stool bulking via the alteration of intestinal microbiota and that koji amazake is a beneficial beverage for individuals with a low defecation frequency.

Supplementary Materials: The following are available online at https://www.mdpi.com/article/10 .3390/jof7090782/s1, Figure S1: The structure of GlcCer contained in koji amazake, Table S1: Background characteristics of the participants, Table S2: Illumina MiSeq sequencing information summary.

Author Contributions: Conceptualization, K.W. and M.H.; methodology, A.K., K.W., N.O., H.G., and M.H.; validation, A.K. and M.H.; formal analysis, M.H.; investigation, K.W., N.O., and H.G.; resources, A.K., T.E., Y.O., A.K.-N., and K.K.; writing-original draft preparation, A.K., T.E., and M.H.; writing-review and editing, A.K. and M.H.; visualization, A.K., T.E., and M.H.; supervision, A.K. and M.H.; project administration, A.K. and M.H. All authors have read and agreed to the published version of the manuscript.

Funding: This research received no external funding.

Institutional Review Board Statement: This study was approved by the ethics committee of Niigata Bio-research Center (Niigata, Japan; approval number IRB2019-BPD-001), and the study was performed in accordance with the guidelines of the Declaration of Helsinki. The study was registered in the University Hospital Medical Information Network (UMIN) Clinical Trials Registry, Japan, under the code UMIN000038520.

Informed Consent Statement: Informed consent was obtained from all participants involved in the study.

Data Availability Statement: The data that support the findings of this study are available from the corresponding author.

Acknowledgments: The authors express their gratitude to TechnoSuruga Laboratory Co., Ltd. (Shizuoka, Japan) for technical assistance.

Conflicts of Interest: This study was funded by Hakkaisan Brewery Co., Ltd., a koji amazake manufacturing company. A.K. is the managing director for production, and research and development. T.E., Y.O., A.K.-N., and K.K. are employees.

\section{References}

1. Campbell-Platt, G. Fermented foods-A world perspective. Food Res. Int. 1994, 27, 253-257. [CrossRef]

2. Afrc, R.F. Probiotics in man and animals. J. Appl. Bacteriol. 1989, 66, 365-378. [CrossRef]

3. Matsumoto, K.; Takada, T.; Shimizu, K.; Moriyama, K.; Kawakami, K.; Hirano, K.; Kajimoto, O.; Nomoto, K. Effects of a probiotic fermented milk beverage containing Lactobacillus casei strain Shirota on defecation frequency, intestinal microbiota, and the intestinal environment of healthy individuals with soft stools. J. Biosci. Bioeng. 2010, 110, 547-552. [CrossRef]

4. Arakawa, C.; Suzuki, S.; Nobuta, Y.; Fu, M.; Suzuki, S.; Sunabori, S.; Suganuma, H. Effects of Lactobacillus Brevis KB290 on Bowel Movement and on Intestinal Environment in Normal Healthy Volunteers with a Tendency toward Constipation: A Randomized, Double-Blind, Placebo-Controlled, Cross-over Trial. J. Nutr. Food 2017, 16, 1-18. [CrossRef]

5. Yaeshima, T.; Takahashi, S.; Matsumoto, N.; Ishibashi, N.; Hayasawa, H.; Iino, H. Effect of Yogurt Containing Bifidobacterium longum BB536 on the Intestinal Environment, Fecal Characteristics and Defecation Frequenc. Biosci. Microflora 1997, 16, 73-77. [CrossRef]

6. Kurahashi, A. Ingredients, Functionality, and Safety of the Japanese Traditional Sweet Drink Amazake. J. Fungi 2021, 7, 469. [CrossRef]

7. Sumiyoshi, K.; Nakao, M. Effect of Amazake Ingestion on Constipation. Jpn. J. Nurs. Art Sci. 2017, 16, 36-40. [CrossRef]

8. Sakurai, M.; Kubota, M.; Iguchi, A.; Shigematsu, T.; Yamaguchi, T.; Nakagawa, S.; Kurahashi, A.; Oguro, Y.; Nishiwaki, T.; Aihara, K.; et al. Effects of Koji amazake and Its Lactic Acid Fermentation Product by Lactobacillus sakei UONUMA on Defecation Status in Healthy Volunteers with Relatively Low Stool Frequency. Food Sci. Technol. Res. 2019, 25, 853-861. [CrossRef]

9. Yamada, K.; Sato-Mito, N.; Nagata, J.; Umegaki, K. Health Claim Evidence Requirements in Japan. J. Nutr. 2008, 138, 1192S-1198S. [CrossRef] [PubMed] 
10. Dimidi, E.; Christodoulides, S.; Fragkos, K.; Scott, S.M.; Whelan, K. The effect of probiotics on functional constipation in adults: A systematic review and meta-analysis of randomized controlled trials. Am. J. Clin. Nutr. 2014, 100, 1075-1084. [CrossRef]

11. Yu, T.; Zheng, Y.-P.; Tan, J.-C.; Xiong, W.-J.; Wang, Y.; Lin, L. Effects of Prebiotics and Synbiotics on Functional Constipation. Am. J. Med. Sci. 2017, 353, 282-292. [CrossRef]

12. Gibson, G.R.; Roberfroid, M.B. Dietary Modulation of the Human Colonic Microbiota: Introducing the Concept of Prebiotics. J. Nutr. 1995, 125, 1401-1412. [CrossRef]

13. Oguro, Y.; Nishiwaki, T.; Shinada, R.; Kobayashi, K.; Kurahashi, A. Metabolite profile of koji amazake and its lactic acid fermentation product by Lactobacillus sakei UONUMA. J. Biosci. Bioeng. 2017, 124, 178-183. [CrossRef]

14. Oguro, Y.; Nakamura, A.; Kurahashi, A. Effect of temperature on saccharification and oligosaccharide production efficiency in koji amazake. J. Biosci. Bioeng. 2018, 127, 570-574. [CrossRef]

15. Unno, T.; Sugawara, M.; Nakakuki, T.; Okada, G. Effect of $\beta$-Glucooligosaccharides on the Human Intestinal Microflora. Bifidobact. Microflora. 1993, 5, 37-50. [CrossRef]

16. Nagura, T.; Muraguchi, H.; Uchino, K.; Aritsuka, T.; Benno, Y. Effects of Ingestion of Raffinose-Rich Soup on the Fecal Flora and Daily Defecation in Humans. J Intestin. Microbiol. 1999, 13, 1-7. [CrossRef]

17. Kohmoto, T.; Fukui, F.; Takaku, H.; Mitsuoka, T. Dose-response Test of Isomaltooligosaccharides for Increasing Fecal Bifidobacteria. Agric. Biol. Chem. 1991, 55, 2157-2159. [CrossRef]

18. Kaneko, T.; Kohmoto, T.; Kikuchi, H.; Shiota, M.; Yatake, T.; Iino, H.; Tsuji, K. Effects of Isomaltooligosaccharides Intake on Defecation and Intestinal Environment in Healthy Volunteers. J. Home Econ. Jpn. 1993, 44, 245-254. [CrossRef]

19. Yasuhiko, F.; Masao, O. Structure of cerebroside in Aspergillus oryzae. Biochim. Et Biophys. Acta (BBA)-Lipids Lipid Metab. 1977, 486, 161-171. [CrossRef]

20. Sakamoto, M.; Skatani, M.; Ferdouse, J.; Hmajima, H.; Tsuge, K.; Nishimukai, M.; Yanagita, T.; Nagao, K.; Mitsukake, S.; Kitagaki, H. Development of a Quantitative Method for the Contents of Glycosylceramide Contained in Japanese Foods Brewed with Koji and Its Application. J. Brew Soc. Jan. 2017, 112, 655-662.

21. Hamajima, H.; Fujikawa, A.; Yamashiro, M.; Ogami, T.; Kitamura, S.; Tsubata, M.; Tan, S.; Matsunaga, H.; Sawada, K.; Kumagai, S.; et al. Chemical Analysis of the Sugar Moiety of Monohexosylceramide Contained in Koji, Japanese Traditional Rice Fermented with Aspergillus. Fermentation 2016, 2, 2. [CrossRef]

22. Hamajima, H.; Matsunaga, H.; Fujikawa, A.; Sato, T.; Mitsutake, S.; Yanagita, T.; Nagao, K.; Nakayama, J.; Kitagaki, H. Japanese traditional dietary fungus koji Aspergillus oryzae functions as a prebiotic for Blautia coccoides through glycosylceramide: Japanese dietary fungus koji is a new prebiotic. SpringerPlus 2016, 5, 1321. [CrossRef]

23. Rodriguez, J.; Neyrinck, A.M.; Zhang, Z.; Seethaler, B.; Nazare, J.-A.; Sánchez, C.R.; Roumain, M.; Muccioli, G.G.; Bindels, L.B.; Cani, P.D.; et al. Metabolite profiling reveals the interaction of chitin-glucan with the gut microbiota. Gut Microbes $2020,12$. [CrossRef]

24. Kurahashi, A.; Nakamura, A.; Oguro, Y.; Watanabe, K.; Ozaki, N.; Goto, H.; Hirayama, M. Beneficial Effects of Koji Amazake in Suppressing the Postprandial Increase in Blood Glucose and Insulin Levels in Healthy Adults. J. Brew Soc. Jan. 2020, 115, 43-53.

25. Fujii, F.; Ozeki, K.; Kanda, A.; Hamachi, M.; Nunokawa, Y. A Simple Method for the Determination of Grown Mycelial Content in Ricekoji using Commercial Cell Wall Lytic Enzyme, Yatalase. J. Brew. Soc. Jpn. 1992, 87, 757-759. [CrossRef]

26. Reissig, J.L.; Storminger, J.L.; Leloir, L. A Modified Colorimetric Method for the Estimation of N-Acetylamino Sugars. J. Biol. Chem. 1955, 217, 959-966. [CrossRef]

27. Ogata, T.; Nakamura, T.; Anjitsu, K.; Yaeshima, T.; Takahashi, S.; Fukuwatari, Y.; Ishibashi, N.; Hayasawa, H.; Fujisawa, T.; Iino, H. Effect of Bifidobacterium Longum BB536 Administration on the Intestinal Environment, Defecation Frequency and Fecal Characteristics of Human Volunteers. Biocsi. Microflora 1997, 16, 53-58. [CrossRef]

28. Saito, T.; Hayakawa, T.; Nakamura, K.; Takita, T.; Suzuki, K.; Innami, S. Fecal Output, Gastrointestinal Transit Time, Frequency of Evacuation and Apparent Excretion Rate of Dietary Fiber in Young Men Given Diets Containing Different Levels of Dietary Fiber. J. Nutr. Sci. Vitaminol. 1991, 37, 493-508. [CrossRef]

29. Lewis, S.J.; Heaton, K.W. Stool Form Scale as a Useful Guide to Intestinal Transit Time. Scand. J. Gastroenterol. 1997, 32, 920-924. [CrossRef]

30. Takahashi, S.; Tomita, J.; Nishioka, K.; Hisada, T.; Nishijima, M. Development of a Prokaryotic Universal Primer for Simultaneous Analysis of Bacteria and Archaea Using Next-Generation Sequencing. PLoS ONE 2014, 9, e105592. [CrossRef]

31. Hisada, T.; Endoh, K.; Kuriki, K. Inter- and intra-individual variations in seasonal and daily stabilities of the human gut microbiota in Japanese. Arch. Microbiol. 2015, 197, 919-934. [CrossRef]

32. Aronesty, E. Comparison of Sequencing Utility Programs. Open Bioinformatics J. 2013, 7, 1-8. [CrossRef]

33. Gordon, A.; Hannon, G.J. FASTQ/A Short-Reads Preprocessing Tools. 2010. Available online: http://hannonlab.cshl.edu/fastx toolkit/ (accessed on 24 August 2020).

34. Caporaso, J.G.; Kuczynski, J.; Stombaugh, J.; Bittinger, K.; Bushman, F.; Costello, E.K.; Fierer, N.; Peña, A.G.; Goodrich, J.K.; Gordon, J.I.; et al. QIIME allows analysis of high-throughput community sequencing data. Nat. Methods 2010, 7, 335-336. [CrossRef]

35. Edgar, R.C.; Haas, B.J.; Clemente, J.C.; Quince, C.; Knight, R. UCHIME improves sensitivity and speed of chimera detection. Bioinformatics 2011, 27, 2194-2200. [CrossRef] 
36. Wang, Q.; Garrity, G.M.; Tiedje, J.M.; Cole, J.R. Naïve Bayesian Classifier for Rapid Assignment of rRNA Sequences into the New Bacterial Taxonomy. Appl. Environ. Microbiol. 2007, 73, 5261-5267. [CrossRef]

37. Kasai, C.; Sugimoto, K.; Moritani, I.; Tanaka, J.; Oya, Y.; Inoue, H.; Tameda, M.; Shiraki, K.; Ito, M.; Takei, Y.; et al. Comparison of the gut microbiota composition between obese and non-obese individuals in a Japanese population, as analyzed by terminal restriction fragment length polymorphism and next-generation sequencing. BMC Gastroenterol. 2015, 15, 1-10. [CrossRef] [PubMed]

38. Hirata, M.; Tsuge, K.; Jayakody, L.N.; Urano, Y.; Sawada, K.; Inaba, S.; Nagao, K.; Kitagaki, H. Structural Determination of Glucosylceramides in the Distillation Remnants of Shochu, the Japanese Traditional Liquor, and Its Production by Aspergillus kawachii. J. Agric. Food Chem. 2012, 60, 11473-11482. [CrossRef]

39. Sugawara, T.; Aida, K.; Duan, J.; Hirata, T. Analysis of glucosylceramides from various sources by liquid chromatography-ion trap mass spectrometry. J. Oleo Sci. 2010, 59, 387-394. [CrossRef] [PubMed]

40. Hijova, E.; Chmelarova, A. Short chain fatty acids and colonic health. Bratisl Lek List. 2007, 108, 354-358.

41. Kruse, H.-P.; Kleessen, B.; Blaut, M. Effects of inulin on faecal bifidobacteria in human subjects. Br. J. Nutr. 1999, 82, 375-382. [CrossRef]

42. Swanson, K.; Grieshop, C.M.; Flickinger, E.A.; Bauer, L.L.; Wolf, B.W.; Chow, J.; Garleb, K.A.; Williams, J.A.; Fahey, G.C. Fructooligosaccharides and Lactobacillus acidophilus Modify Bowel Function and Protein Catabolites Excreted by Healthy Humans. J. Nutr. 2002, 132, 3042-3050. [CrossRef] [PubMed]

43. Hess, J.; Wang, Q.; Gould, T.; Slavin, J. Impact of Agaricus bisporus Mushroom Consumption on Gut Health Markers in Healthy Adults. Nutrients 2018, 10, 1402. [CrossRef]

44. Asai, S.; Miyachi, H. Evaluation of skin-moisturizing effects of oral or percutaneous use of plant ceramides. Rinsho byori. Jpn. J. Clin. Pathol. 2007, 55, 209-215.

45. Uchiyama, T.; Nakano, Y.; Ueda, O.; Mori, H.; Nakashima, M.; Noda, A.; Ishizaki, C.; Mizoguchi, M. Oral Intake of Glucosylceramide Improves Relatively Higher Level of Transepidermal Water Loss in Mice and Healthy Human Subjects. J. Health Sci. 2008, 54, 559-566. [CrossRef]

46. Miyagawa, M.; Fujikawa, A.; Nagadome, M.; Kohama, K.; Ogami, T.; Kitamura, S.; Kitagaki, H. Glycosylceramides Purified from the Japanese Traditional Non-Pathogenic Fungus Aspergillus and Koji Increase the Expression of Genes Involved in Tight Junctions and Ceramide Delivery in Normal Human Epidermal Keratinocytes. Fermentation 2019, 5, 43. [CrossRef]

47. Yoshimi, A.; Miyazawa, K.; Abe, K. Cell wall structure and biogenesis in Aspergillus species. Biosci. Biotechnol. Biochem. 2016, 80, 1700-1711. [CrossRef]

48. Ley, R.E.; Turnbaugh, P.J.; Klein, S.; Gordon, J.I. Microbial Ecology: Human Gut Microbes Associated with Obesity. Nature 2006, 444, 1022-1023. [CrossRef]

49. Schwiertz, A.; Taras, D.; Schäfer, K.; Beijer, S.; Bos, N.A.; Donus, C.; Hardt, P.D. Microbiota and SCFA in Lean and Overweight Healthy Subjects. Obesity 2010, 18, 190-195. [CrossRef]

50. Fernandez-Raudales, D.; Hoeflinger, J.L.; Bringe, N.A.; Cox, S.B.; Dowd, S.; Miller, M.J.; De Mejia, E.G. Consumption of different soymilk formulations differentially affects the gut microbiomes of overweight and obese men. Gut Microbes 2012, 3, 490-500. [CrossRef]

51. Kassaian, N.; Feizi, A.; Rostami, S.; Aminorroaya, A.; Yaran, M.; Amini, M. The effects of 6 mo of supplementation with probiotics and synbiotics on gut microbiota in the adults with prediabetes: A double blind randomized clinical trial. Nutrition 2020, 79-80, 110854. [CrossRef] 